\title{
Topological superconductivity by doping symmetry-protected topological states
}

\author{
Shang-Qiang Ning, ${ }^{1}$ Zheng-Xin Liu $\odot,{ }^{2, *}$ and Hong-Chen Jiang ${ }^{3, \dagger}$ \\ ${ }^{1}$ Institute for Advanced Study, Tsinghua University, Beijing 100084, China \\ ${ }^{2}$ Department of Physics, Renmin University of China, Beijing 100872, China \\ ${ }^{3}$ Stanford Institute for Materials and Energy Sciences, SLAC and Stanford University, 2575 Sand Hill Road, \\ Menlo Park, California 94025, USA
}

(Received 27 July 2019; revised manuscript received 1 April 2020; accepted 16 April 2020; published 18 May 2020)

\begin{abstract}
We propose an exotic scenario in which topological superconductivity can emerge by doping strongly interacting fermionic systems whose spin degrees of freedom form a bosonic symmetry protected topological (SPT) state. Specifically, we study a one-dimensional (1D) example where the spin degrees of freedom form a spin-1 Haldane phase. Before doping, the charge and spin degrees of freedom are both gapped. Upon doping, the charge channel becomes gapless and is described by a $c=1$ compactified bosonic conformal field theory (CFT), while the spin channel remains gapped and still form a bosonic SPT state. Interestingly, an instability toward $p$-wave topological superconductivity is induced, coexisting with the symmetry protected spin edge modes that are inherited from the Haldane phase. This scenario is confirmed by density-matrix renormalization group simulation of a concrete lattice model, where we find that topological superconductivity is robust against interactions. We further show that by stacking doped Haldane phases an exotic 2D anisotropic superconductor can be realized, where the boundaries transverse to the chain direction are either gapless or spontaneously symmetry broken due to the Lieb-Schultz-Mattis (LSM) anomaly.
\end{abstract}

DOI: 10.1103/PhysRevResearch.2.023184

\section{INTRODUCTION}

The interplay between symmetry and topology plays an important role in modern condensed matter physics, and leads to the discovery and classification of various types of exotic (gapped) phases of matter, among which are the symmetryprotected topological (SPT) phases [1]. Characteristic SPT phases include the topological insulators [2-4], Haldane phase [5,6] and bosonic integer quantum Hall liquids [7]. In SPT phases, the bulk excitations are gapped and unfractionalized while the edge states can be either gapless or topologically ordered if the symmetry is not broken [8].

Bosonic SPT (bSPT) phases are (partially) classified by group cohomology theory $[9,10]$. However, the classification of fermionic SPT (fSPT) phases is rather involved. Noninteracting fermionic topological phases are classified by $\mathrm{K}$ theory $[11,12]$. When interactions are turned on, the classes of fSPT phases will generally be changed. A typical example is one-dimensional (1D) time-reversal-invariant topological superconductors, where interactions reduce the noninteracting $Z$ classes into $Z_{8}[13,14]$. A systematic classification of interacting fSPT phases is relatively difficult, while remarkable

\footnotetext{
*liuzxphys@ ruc.edu.cn

†hcjiang@stanford.edu

Published by the American Physical Society under the terms of the Creative Commons Attribution 4.0 International license. Further distribution of this work must maintain attribution to the author(s) and the published article's title, journal citation, and DOI.
}

progress has been made recently [15-18]. Roughly speaking, interacting fSPT phases can be divided into three types [19]: (I) those that are adiabatically connected to noninteracting fermion SPT phases; (II) those that are adiabatically connected to bosonic SPT phases; (III) those that can only be realized in interacting fermionic systems. While type-I and type-III fSPT phases only exist in pure fermionic systems, the type-II phases can be obtained by freezing the fermionic charge degrees of freedom to form Mott insulators (or by enforcing the fermions to tightly bind into bosonic molecules) and then letting the bosonic spin (or molecule) degrees of freedom form a bSPT phase [20]. Some of the type-II fSPT phases can also be obtained by stacking a band insulator with a bSPT phase. In other words, such of phases can be realized by embedding a bSPT phase in fermionic system and can be called bSPT-embedded phases [19].

In this paper, we investigate the physical consequences of doping holes into the type-II fSPT insulators. We expect that the holes in such systems can exhibit nontrivial charge behavior, which drives the system into a superconductor. This is illustrated by a concrete example, where the embedded bSPT phase is the spin-1 Haldane phase whose dangling spin-1/2 edge states are protected by time reversal, or $\mathrm{SO}(3)$ (or its subgroup $\mathbb{Z}_{2} \times \mathbb{Z}_{2}$ ) symmetry [1,21]. The way we embed the spin-1 Haldane phase into a nontrivial fSPT phase is to introduce three species of spinless fermions $\psi_{x}, \psi_{y}, \psi_{z}$ on each site [20]. We show that the Haldane phase at $1 / 3$ filling has a topological superconducting instability upon doping. This scenario is confirmed by large-scale density-matrix renormalization group (DMRG) [22] simulations. We further conclude that the superconducting instability can be turned 
into a $p$-wave superconductor by coupling the $1 \mathrm{D}$ wires to from a $2 \mathrm{D}$ lattice.

\section{CONTINUOUS FIELD THEORY}

Similar to [20], we consider a fermion ladder with three species $\psi_{x}, \psi_{y}, \psi_{z}$, whose Hamiltonian is $H=H_{0}+H_{I}$. Here $H_{0}$ is the noninteracting part $H_{0}=-t \sum_{\alpha, i} \psi_{\alpha, i}^{\dagger} \psi_{\alpha, i+1}+$ H.c. and $H_{I}$ is the interacting part which will be specified later. Different $H_{1}$ can realize different phases. The system contains time reversal symmetry, global U(1) symmetry generated by the total fermion charge $\hat{n}=\sum_{\alpha, i} \psi_{\alpha, i}^{\dagger} \psi_{\alpha, i}$, and $\operatorname{SO}(3)$ symmetry (or its subgroup $\mathbb{Z}_{2} \times \mathbb{Z}_{2}$ ) which mixes the three species. Assuming that the interaction is not strong, we can treat $H_{I}$ as perturbations and use the bosonization technique to analyze the physical consequences. By adjusting the interactions $H_{I}$ and the filling, several interesting phases can be obtained.

The unperturbed part $H_{0}$ is described by three isotropic Luttinger liquids with action

$$
H_{0}=\frac{v_{F}}{2 \pi} \int d x \sum_{\alpha=x, y, z}\left(\partial_{x} \phi_{\alpha}\right)^{2}+\left(\partial_{x} \theta_{\alpha}\right)^{2},
$$

where $\phi_{\alpha}$ are the bosonized fields and $\theta_{\alpha}$ are their dual fields which satisfy $\left[\partial_{x} \phi_{\alpha}(x), \theta_{\beta}\left(x^{\prime}\right)\right]=i \pi \delta_{\alpha, \beta} \delta\left(x-x^{\prime}\right)$. The spatial derivative of $-\pi \phi_{\alpha}$ (or $\pi \theta_{\alpha}$ ) is the density(or current) of the fermion $\psi_{\alpha}$. We transform the original bases of these fields into one charge channel and two spin channels, namely, $\phi_{c}=\sqrt{1 / 3}\left(\phi_{x}+\phi_{y}+\phi_{z}\right), \phi_{s_{1}}=\sqrt{2 / 3} \phi_{x}+\sqrt{1 / 6}\left(\phi_{y}+\phi_{z}\right)$, and $\phi_{s_{2}}=\sqrt{1 / 2}\left(\phi_{z}-\phi_{y}\right)$. In this way, $\partial_{x} \phi_{c}, \partial_{x} \phi_{s_{1,2}}$ stand for the total charge and spin densities, respectively. For later convenience, we define the fields $\Phi_{1}=\phi_{x}-\phi_{y}$ and $\Phi_{2}=$ $\phi_{y}-\phi_{z}$ as well, which do not involve the total charge degree of freedom. The dual fields $\theta_{c}, \theta_{s_{1,2}}$ and the fields $\Theta_{1}, \Theta_{2}$ are defined similarly.

The Luttinger liquid in (1) can be gapped out by condensing some bosonic fields (with suitably chosen $H_{I}$ ). There are several ways; for instance, one can condense (1) $\phi_{c}$ and $\phi_{s_{1,2}}$, or (2) $\phi_{c}$ and $\theta_{s_{1,2}}$, or (3) $\theta_{c}$ and $\phi_{s_{1,2}}$, or (4) $\theta_{c}$ and $\theta_{s_{1,2}}$. Different condensation patterns result in different phases. To obtain a bSPT embedded phase, we require that the spin degrees of freedom be gapped in a nontrivial way. To achieve this, we condense the $2 \Theta_{1}, 2 \Theta_{2}$ fields. ${ }^{1}$ The physical meaning of $2 \Theta_{1}, 2 \Theta_{2}$ is given by $e^{i 2 \Theta_{1}(x)} \sim \Delta_{x}^{\dagger}(x) \Delta_{y}(x)$ and $e^{i 2 \Theta_{2}(x)} \sim$ $\Delta_{y}^{\dagger}(x) \Delta_{z}(x)$, where $\Delta_{\alpha}=\psi_{R \alpha(x)} \psi_{L \alpha}(x+a), \alpha=x, y, z$. The charge gap can be opened by Umklapp process which scatters the three left-moving fermions into the three right-moving fermions and vice versa. This process keeps the $U(1)$ and $\mathrm{SO}(3)$ symmetries unbroken, but it requires that the fraction of the particle number filling be commensurate, such as $1 / 3$ $[23,24]$. As illustrated in Appendix A, the condensation of

\footnotetext{
${ }^{1}$ The condensation of $2 \Theta_{1,2}$ is obviously invariant under $\mathbb{Z}_{2} \times \mathbb{Z}_{2}$, a subgroup of $\mathrm{SO}(3)$. Though the $\mathrm{SO}(3)$ symmetry in not apparent in Abelian bosonization, this subgroup can also protect the same Haldane phase. We further argue that this condensation can indeed be $\mathrm{SO}(3)$ invariant since it can come from some $\mathrm{SO}(3)$ invariant interactions.
}

$\phi_{c}$ and $2 \Theta_{1}, 2 \Theta_{2}$ at $1 / 3$ filling indeed results in fully gapped ground states whose degeneracy is 4 under open boundary condition, and the edge modes carry nontrivial projective representation of the $\mathrm{SO}(3)$. This state is adiabatically connected to the spin-1 Haldane chain.

Once the system is slightly doped away from $1 / 3$ filling, the Umklapp process will be suppressed and hence the $\phi_{c}, \theta_{c}$ fields become gapless and are characterized by the $c=1$ compactified bosonic conformal field theory (CFT)

$$
H_{c}=\frac{1}{2 \pi} \int d x\left[u_{c} K_{c}\left(\partial \theta_{c}\right)^{2}+\frac{u_{c}}{K_{c}}\left(\partial \phi_{c}\right)^{2}\right],
$$

where $u_{c}$ is the renormalized fermi velocity and $K_{c}$ the radius parameter. In the noninteracting limit, $u_{c}=v_{F}$ and $K_{c}=1$. Meanwhile, due to spin-charge separation, the condensation of $2 \Theta_{1,2}$ is unaffected and the spin channels are still gapped, leaving the spin-edge modes at open boundaries untouched. In this case, the dominant correlation function is of the $\mathrm{SO}(3)$-singlet pairing $\Delta_{\mathrm{SC}}=\sum_{\alpha=x, y, z} \Delta_{\alpha}$, which has odd parity under fermion exchanging (see Appendix B). In bosonized fields, this quantity is written as $\Delta_{\mathrm{SC}}=$ $\frac{e^{i \frac{2}{\sqrt{3}} \theta_{c}}}{2 \pi a}\left[e^{i \sqrt{\frac{8}{3}} \theta_{s_{1}}}+2 e^{i \sqrt{\frac{2}{3}} \theta_{s_{1}}} \cos \left(\sqrt{2} \theta_{s_{2}}\right)\right]+\cdots$, where the cutoff $a$ is the lattice constant. Since $\theta_{s_{1}}$ and $\theta_{s_{2}}$ are condensed and $\theta_{c}$ is gapless in the doped fermionic Haldane phase, $\left\langle\Delta_{\mathrm{SC}}(r+\right.$ $\left.\left.r_{0}\right) \Delta_{\mathrm{SC}}\left(r_{0}\right)\right\rangle \propto 1 / r^{\frac{2}{3 K_{c}}}$, where $K_{c}$ is the Luttinger parameter of the charge channel.

On the other hand, if we condense the $2 \Phi_{1}$ and $2 \Phi_{2}$ fields, then the degenerate spin-edge modes go away, and a trivial gapped phase is obtained. In this case, under light doping $\phi_{c}$ is gapless and is still characterized by (2), where all superconducting correlation functions are exponentially decaying while the total particle density wave correlation becomes power-law decaying (see Appendix B).

Above we have shown that the condensation of $\phi_{c}, 2 \Theta_{1}, 2 \Theta_{2}$ at $1 / 3$ filling gives rise to the Haldane phase. When doped away from commensurate filling, the system shows an instability toward an $\mathrm{SO}(3)$-invariant odd-parity superconductivity. The above scenario can be realized by adding several simple interactions to the system. A natural interaction is the antiferromagnetic Heisenberg exchange. To drive the system into a Mott insulator, a Hubbard interaction is also plausible. Notice that in above discussion we have assumed that the interactions are not strong, otherwise they may be an obstacle to the hopping of charge and lead to phase separation (see below for more details).

\section{LATTICE MODEL}

The discussion above leads us to study the following interaction (together with the aforementioned isotropic hopping terms $\left.H_{0}\right)$ :

$$
H_{I}=J \sum_{i} \mathbf{S}_{i} \cdot \mathbf{S}_{i+1}+U \sum_{i}\left(n_{i}-1\right)^{2},
$$

where $n_{i}=\sum_{\alpha} \psi_{\alpha, i}^{\dagger} \psi_{\alpha, i}$ and $S_{i}^{\alpha}=i \epsilon^{\alpha \beta \gamma} \psi_{\beta, i}^{\dagger} \psi_{\gamma, i}$ (these spin operators satisfy the $\operatorname{su}(2)$ commutation relation if the constraint $n_{i}=1$ is satisfied). When $J \neq 0$, the model has $\operatorname{SO}(3)$ symmetry which is enlarged to $\mathrm{SU}(3)$ in the case $J=0$ (in 
the following, we assume $J>0$ ). Now we fix the filling at $1 / 3-\delta$ where $\delta$ denotes the doping density.

\section{A. Strong coupling limit}

When the filling is $\frac{1}{3}$ and when $U \gg J>t>0$, the system tends to occupy one fermion per site. On-site charge fluctuations are strongly suppressed and the model becomes a pure spin system, i.e., the spin-1 Heisenberg model [20] which belongs to the Haldane phase. Perturbations can be added to the system without destroying the Haldane phase as long as time reversal or $Z_{2} \times Z_{2}$ symmetry is unbroken. However, in the present work we do not discuss such perturbations in detail.

\section{B. Weak coupling limit}

When the interaction strength is much smaller than $t$, we can treat the interactions perturbatively. Then above Hamiltonian decouples into two channels in bosonization terminology, i.e., the charge channel and spin channel. ${ }^{2}$ We first consider the charge channel, $H_{c}^{\prime}=H_{c}+H_{u}$ where $H_{c}$ is (2) with $K_{c}=1+\frac{a}{2 \pi v_{F}}\left[8 \cos \left(2 k_{F} a\right) J-8 U\right]$ and $k_{F}=\pi / 3-\pi \delta$ and $H_{u}$ represents the Umklapp process term for $\delta=0$ $[23,24]$. Without doping, the Umklapp process from higher order perturbation can cause $\phi_{c}$ to condense such that the charge channel opens a gap. ${ }^{3}$ At not very large doping, i.e., $-1 / 6<\delta<1 / 12, \cos \left(2 k_{F} a\right)<0$ and then $K_{c}<1$ for positive $2 U+2 \cos \left(2 k_{F} a\right) J$. However, this process is immediately suppressed by nonzero but small $\delta$.

Now we consider the spin channel,

$$
\begin{aligned}
H_{s}= & \frac{1}{2 \pi} \int d x \sum_{\beta=s_{1}, s_{2}}\left[u_{\beta} K_{\beta}\left(\partial \theta_{\beta}\right)^{2}+\frac{u_{\beta}}{K_{\beta}}\left(\partial \phi_{\beta}\right)^{2}\right] \\
& +g\left[\cos \left(\sqrt{2} \phi_{s_{2}}-\sqrt{6} \phi_{s_{1}}\right)+\cos \left(\sqrt{2} \phi_{s_{2}}+\sqrt{6} \phi_{s_{1}}\right)\right. \\
& \left.+\cos \left(2 \sqrt{2} \phi_{s_{2}}\right)\right]+h\left[\cos \left(\sqrt{2} \theta_{s_{2}}-\sqrt{6} \theta_{s_{1}}\right)\right. \\
& \left.+\cos \left(\sqrt{2} \theta_{s_{2}}+\sqrt{6} \theta_{s_{1}}\right)+\cos \left(2 \sqrt{2} \theta_{s_{2}}\right)\right],
\end{aligned}
$$

where $K_{s_{1}}=K_{s_{2}}=1+\frac{a}{2 \pi v_{F}}\left[4 U-4 \cos \left(2 k_{F} a\right) J\right]=K_{s}$. The one-loop RG equations are

$$
\frac{d y_{g}}{d l}=\left(2-\Delta_{g}\right) y_{g}-y_{g}^{2}, \quad \frac{d y_{h}}{d l}=\left(2-\Delta_{h}\right) y_{h}-y_{h}^{2},
$$

where we have set $y_{g, h}=g(h) / 2 \pi v_{F}$ and $\Delta_{g}=2 K_{s}$, and $\Delta_{h}=2 / K_{s}$ are the scaling dimensions of the $g$ and $h$ interaction terms.

\footnotetext{
${ }^{2}$ One bosonization study of a model similar to (3) in Ref. [25] is closely related to this but also with a significant difference: filling (Ref. [25] focuses on 1/3 filling and we mainly focus on doping away from $1 / 3$ filling) and bare interactions (the pairing interaction $V$ in Ref. [25]) are only part of the Heisenberg-like interaction $J$ in our paper.

${ }^{3}$ Reference [23] concluded that finite critical interaction strength is needed for the Umklapp process to open the charge gap. However, careful numerical calculation in Ref. [24] suggests that the critical interaction strength is approaching zero.
}

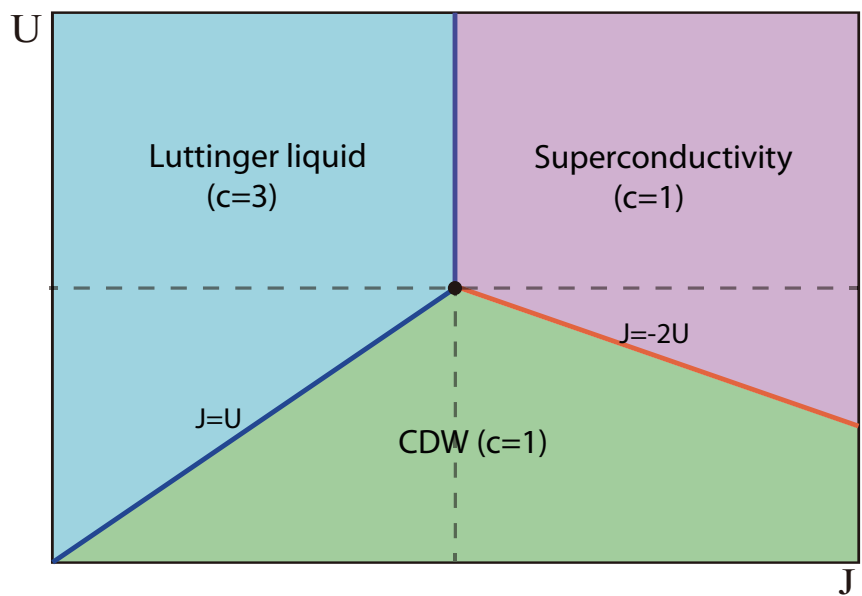

FIG. 1. Bosonization phase diagram with light doping for the Hamiltonian with interaction (3). $c$ represents the central charge. The charge channel is always gapless with $c=1$. The dominant instability of the red regime is superconductivity which is $\Delta_{\mathrm{SC}}=$ $\sum_{\alpha} \psi_{\alpha}(x) \psi_{\alpha}(x+a)$ while that of the green regime is density wave instability which is $n(x)=\sum_{\alpha} n_{\alpha}(x)$. The Luttinger liquid is gapless in both spin and charge channels.

For simplicity we assume the doping is small such that $k_{F} \simeq \pi / 3, \cos \left(2 k_{F} a\right) \simeq-1 / 2$. (For general doping $\delta$ satisfying $-1 / 6<\delta<1 / 12$, the following discussion is still valid.) From the perturbative RG flow of the above equations we obtain the whole phase diagram shown in Fig. 1. We are especially interested in the superconductivity regime with $J>$ 0 and $2 U+J>0$ for light doping (the red area in Fig. 1). In this case $\Delta_{g}>2, \Delta_{h}<2$, thus, at the tree level, $\cos \left(2 \phi_{\alpha}-\right.$ $\left.2 \phi_{\beta}\right)$ are irrelevant while $\cos \left(2 \theta_{\alpha}-2 \theta_{\beta}\right)$ are relevant. Under the one-loop approximation, the above RG equations indicate that $y_{g}$ flows to zero while $y_{h}$ flows to the strong coupling limit. Here the initial values of $g$ and $h$ are $4 U-4 J$ and $-6 J$, respectively. On the other hand, in the regime $U>J$, $2 U+J<0$, and $U<0$ (i.e. the green area in Fig. 1), $y_{g}$ flows to the strong coupling limit. Notice that $y_{h}\left(y_{g}\right)$ going to the strong coupling limit indicates the condensation of $2 \Theta_{1,2}\left(2 \Phi_{1,2}\right)$. In the rest regime, nothing is condensed and the system is still a Luttinger liquid.

\section{NUMERICAL RESULTS}

To verify above field theory analysis, now we determine the ground state phase diagram of the lattice model (3) by extensive DMRG [22] simulations. We focus on a system with open boundary condition. The total number of sites is $N=3 L$, where $L$ is the length and 3 corresponds to the three-leg ladder. The number of fermions would be $N_{e}=L$ at $1 / 3$ filling and the doped hole concentration is defined as $\delta=N_{h} / N$, where $N_{h}=L-N_{e}$ is the number of doped holes, which equals 0 at $1 / 3$ filling. In our calculation, we focus on the hole concentration $\delta=5 \%$. We set $t=1$ as an energy unit and keep up to $m=4000$ states in each DMRG block with truncation error $\epsilon<10^{-8}$ for system length up to $L=240$. This leads to excellent convergence for our results when extrapolated to the $m=\infty$ limit. 


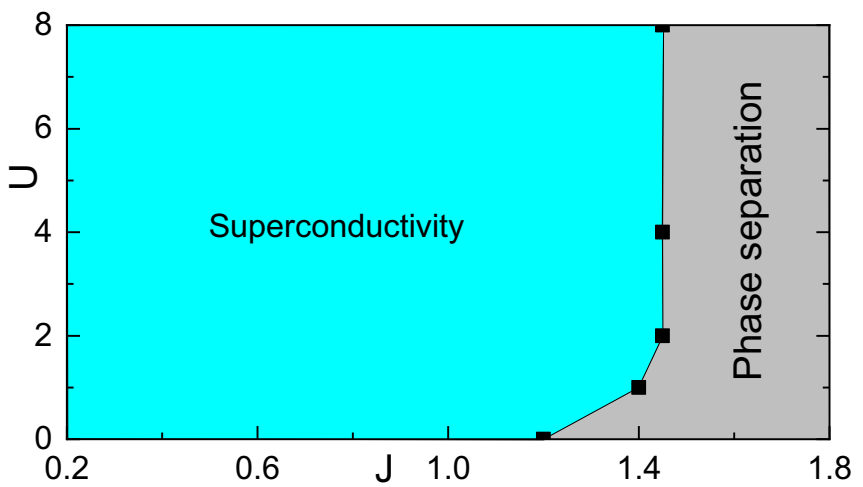

FIG. 2. Ground state phase diagram of the model in Eq. (3) at doping level $\delta=5 \%$ as a function of $U$ and $J$.

Our primary results are summarized in the phase diagram in Fig. 2 at doping level $\delta=5 \% .{ }^{4}$ Two distinct phases, including the superconducting (SC) phase and phase separation (PS) with central charges $c=1$ and 0 respectively (see Appendix C) are found with the increase of $J / t$. The bulk of the phase diagram is occupied by the former one which is dominated by the $\mathrm{SO}(3)$-singlet odd-parity superconducting correlation. While in the region of phase separation, the doped holes are accumulated in a small region close to the open ends while the bulk of the system is still in the fully gapped SPT phase.

Here we are mainly interested in the superconducting region, for which we show an example for a characteristic set of parameters $J=1.0$ and $U=4$ in Fig. 3. The spinspin correlation function $F(r)=\left\langle\mathbf{S}_{i} \cdot \mathbf{S}_{x+r}\right\rangle \sim e^{-r / \xi_{s}}$ decays exponentially, where $r$ is the distance between two sites and $\xi_{s}$ is the correlation length, indicating that the spin is gapped and disordered. In contrast, the density-density correlation, defined as $D(r)=\langle\tilde{n}(x) \tilde{n}(x+r)\rangle$ where $\tilde{n}=n_{i}-1$, is powerlaw decaying, $D(r) \sim r^{-K_{c}}$ (see Fig. 3), indicating that the charge mode is gapless, which is in the $c=1$ Luttinger liquid (see Appendix C). Furthermore, we find that the correlation of the previously mentioned $\mathrm{SO}(3)$-singlet pairing operator $\Delta_{\mathrm{SC}}$, namely $\Phi(r)=\left\langle\Delta_{\mathrm{SC}}^{+}(x) \Delta_{\mathrm{SC}}(x+r)\right\rangle$, also decays as a power law, $\Phi(r) \sim r^{-K_{\mathrm{SC}}}$. Interestingly, our results suggest that $\Phi(r)$ is the dominant correlation as $K_{\mathrm{SC}}<K_{c}$. For instance, $K_{\mathrm{SC}} \sim$ 1.34 and $K_{c} \sim 1.80$ for the results in Fig. 3. We have also calculated other cases, with $J=0.3, U=4$ and $J=1.0$, $U=0.3$, and found that the exponents $K_{\mathrm{SC}}=0.82,1.73$ are smaller than those of $K_{c}=1.79,1.77$, respectively. These results verified our previous prediction that the system has a instability towards $p$-wave superconductivity. ${ }^{5}$

\footnotetext{
${ }^{4}$ We have also studied the $J<0.2$ region using DMRG. While our results are consistent with those of $J \geqslant 0.2$ region with superconductivity, it has visually bigger finite size effect. Hence, we will only show our results in the $J \geqslant 0.2$ region in this work.

${ }^{5}$ Strictly speaking, the doped state with pairing correlation has a instability toward superfluidity. We consider this phase as superconducting in the sense that it can be realized in an electron system. The spinless fermion can be considered as a spin-polarized electron, and hence the Haldane chain is realized by a three-leg electron ladder.
}

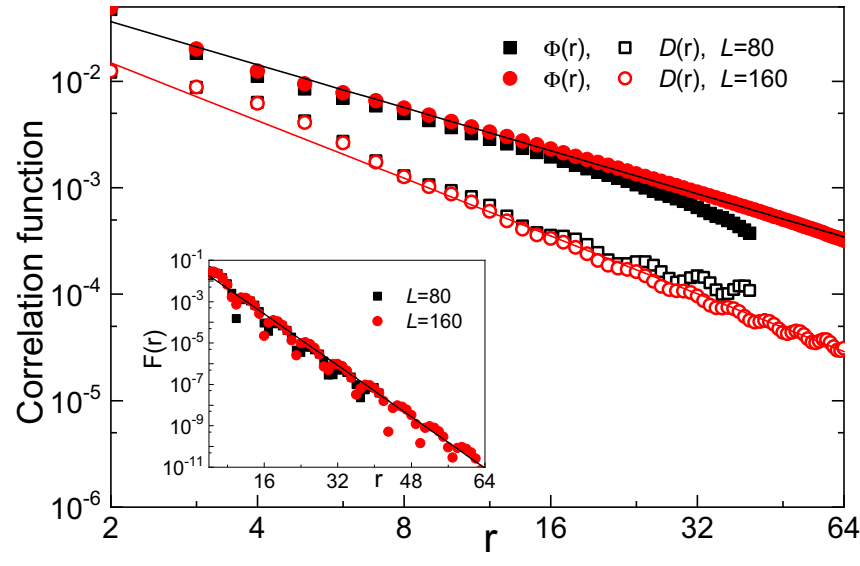

FIG. 3. Superconducting $\Phi(r)$ and density-density $D(r)$ correlation functions for the model in Eq. (3) at doping level $\delta=5 \%$ with $U=4$ and $J=1.0$ in a double-logarithmic plot. The solid lines are fits to $\Phi(r) \sim r^{-K_{\mathrm{SC}}}$ (black) and $D(r) \sim r^{-K_{c}}$ (red), respectively. The inset is the spin-spin correlation function $F(r)$ in semilogarithmic scales for the system, and the solid line is the fit to $F(r) \sim e^{-r / \xi_{s}}$.

To verify the coexistence of the superconductivity and the spin- $1 / 2$ edge modes, we calculate the entanglement spectrum (ES), which is defined as the set of eigenvalues of the reduced density matrix in the ground state $\rho_{A} \equiv \operatorname{Tr}_{B}|\Psi\rangle\langle\Psi|$, with $A$ being a subsystem and $B$ the rest part. Here we use the real space ES with open boundary condition (OBC), where the subsystem $A$ is chosen to be half of the chain with $N / 2$ sites. The lowest level of ES in the superconducting phase is always twofold degenerate, as shown in Fig. 4, which is consistent with the fact that there are two spin- $1 / 2$ edge modes at the open ends. Although the charge channel is gapless, and so its ES would be continuous in the thermodynamic limit, for a finite-sized system the ES contributed from the charge channel (i.e., a $c=1$ compactified bosonic CFT) is discrete and nondegenerate. Therefore, the degeneracy in ES comes from the SPT state in the spin channel.

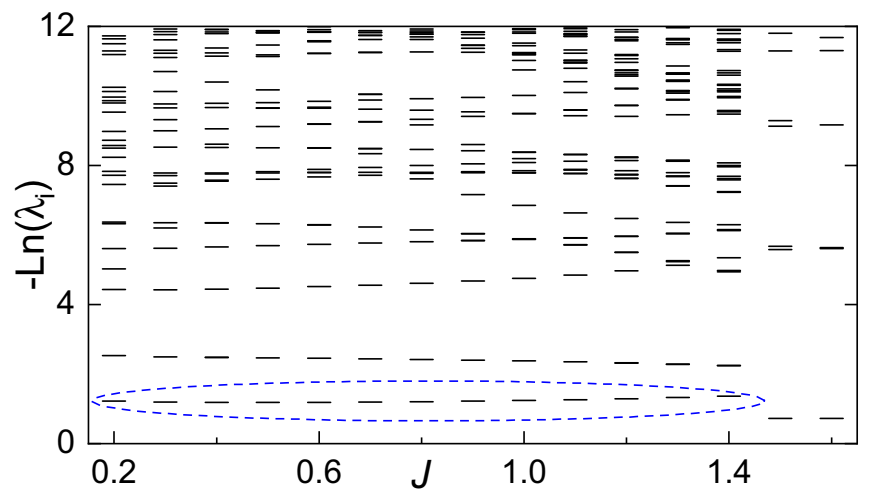

FIG. 4. The 100 lowest levels of the entanglement spectrum $-\ln \left(\lambda_{i}\right)$ for system of length $L=160$ at $\delta=5 \%$ and $U=4$ as a function of $J$, where $\lambda_{i}$ are the eigenvalues of the reduced density matrix. The two largest eigenvalues surrounded by the dashed blue oval are degenerate. 
When the exchange interaction is strong, the system undergoes a phase separation whose central charge becomes zero. This can be understood as the competition between the kinetic energy of the holes and the exchange energy of the spins. When $J$ is large enough, the exchange wins. As a consequence, the holes accumulate at the boundary and the system forms a phase separation (see Appendix C).

\section{2D $p$-WAVE SUPERCONDUCTOR BY COUPLED WIRES}

Here we consider the stacking of the doped chains which have superconducting instability. We label the arrays of the chains by $j$ with $j \in Z$, and use $\left\{\theta_{s_{1}, j}, \theta_{s_{2}, j}, \theta_{c, j}\right\}$ (similarly for the $\phi$ fields) to label the bosonized fields of the $j$ th chain. Without inter-chain coupling, the doped Haldane-embedded chains are independent, thus $\theta_{s_{1}, j}, \theta_{s_{2}, j}$ are condensed and $\theta_{c, j}$ are gapless for all $j \in Z$. We assume that the condensate of $\left\{\theta_{s_{1}, j}, \theta_{s_{2}, j}\right\}$ is unaffected when weak interchain couplings are turned on. The charge channel can be gapped out in several ways; one interesting way is adding mass terms $\cos \left[2 \sqrt{3}\left(\theta_{c, j}-\theta_{c, j+1}\right)\right]$ [26] and tuning them to be relevant. ${ }^{6}$ As a result, the interchain charge fields $\theta_{c, j}-\theta_{c, j+1}$ are locked. In the thermodynamic limit, the total charge field $\theta_{c}=\sum_{j} \theta_{c, j}$ may condense at a specific value, which leads to the spontaneous breaking of the total $\mathrm{U}(1)$ charge symmetry and the emergence of long-range $p$-wave superconductivity. In such a state, the correlation of any interchain pairing order parameters, e.g., $\psi_{x, j} \psi_{x, j+1}$ must be be exponentially decaying since they contains the $\phi_{s_{1,2}}$ fields. Since the $\theta_{s_{1,2}, i}$ fields in each chain are still condensed, the spin $1 / 2$ edge modes are expected to be robust at the chain-ends. If there is no interchain interaction or hopping, these spin- $1 / 2$ edge modes would result in huge degeneracy. If the intensity of interchain interactions is much smaller than the spin gap, then these edge states will either form gapless dispersion or lift the degeneracy by spontaneous breaking of the translation symmetry from the LSM theorem $[27,28]$. We believe that there exists a parameter window for the gapless edge, which may be described by the SU(2) 1 Wess-Zumino-Witten model up to marginal deformations. As a consequence, we find that such an array of charge-doped Haldane-embedded fSPT chains could lead to the coexistence of superconductivity and gapless edge modes. We can also see that the $p+i p$ superconductivity cannot be realized without breaking the condensation of $\theta_{s_{1}, j}, \theta_{s_{2}, j}$. We leave the realization of this 2D chiral topological superconductivity for future study.

\section{SUMMARY AND DISCUSSION}

We study the interplay between the charge fluctuations and nontrivial topology in the spin degrees of freedom in the interacting fermionic SPT phase. Similarly to the proposal that

\footnotetext{
${ }^{6}$ The scaling dimension of these mass terms is $3 / K_{c, j}+3 / K_{c, j+1}$ where $K_{c, j}$ is the Luttinger parameter of the charge channel in the $j$ th chain. To make these mass terms relevant, $K_{c, j}$ must be larger than 3 . To this end, we have to add more interactions in each chain which only contains the forward scattering process to renormalize $K_{c, j}$.
}

doped spin liquids can give rise to high temperature superconductivity [29], here we demonstrate another exotic scenario in which doping the interacting fermionic SPT insulators can also lead to nontrivial superconductivity. We illustrate this possibility by a $1 \mathrm{D}$ example: i.e., doping the Haldane-phaseembedded fSPT phase away from $1 / 3$ filling can result in nontrivial superconducting instability in a certain parameter regime. We further show that stacking such superconducting wires can lead to anisotropic 2D $p$-wave superconductivity coexisting with symmetry-protected gapless edge modes in the transverse direction.

The underlying picture is that the $\mathrm{U}(1)$ charge conservation symmetry in the type-II fSPT insulators, which plays no role in protecting its topological features in the spin channel, can be spontaneously broken by tuning the interactions and the filling fraction. Furthermore, the emergence of superconductivity does not affect the topological nature of the spin degrees of freedom. Therefore, an exotic superconductor is obtained. We believe that such a scenario of obtaining unconventional superconductivity can be generalized in more general settings, especially in two- and higher-dimensional type-II fSPT insulators.

An essential ingredient for the emergent superconductivity in our examples is that the charge fluctuation should not be too weak comparing to the gap in the SPT state formed by spin degrees of freedom. Although the topological properties of the SPT phases can be clearly seen in the topological limit (where the gap is set to infinity), our study suggests that away from the topological limit the competition from other degrees of freedom may also yield interesting new physics in the SPT phases.

\section{ACKNOWLEDGMENTS}

S.-Q.N. thanks Meng Cheng and Zixiang Li for insightful discussions. S.-Q.N. and Z.-X.L. are supported by the Ministry of Science and Technology of China (Grant No. 2016YFA0300504), NSFC (Grants No. 11974421 and No. 11574392), and the Fundamental Research Funds for the Central Universities and the Research Funds of Renmin University of China (No. 19XNLG11). S.-Q.N. is also thankful of support from NSFC 11574172. H.-C.J. is supported by the Department of Energy, Office of Science, Basic Energy Sciences, Materials Sciences and Engineering Division, under Contract No. DE-AC02-76SF00515. Parts of the computing for this project were performed on the Sherlock cluster.

\section{APPENDIX A: GROUND STATE DEGENERACY OF HALDANE PHASE WITH DIFFERENT BOUNDARY CONDITIONS}

In this section, we will show that when $2 \Theta_{1}$ and $2 \Theta_{2}$ condense, the spin channel will be gapped. The ground state is fourfold degeneracy (which can be decomposed into a singlet and a triplet) under open boundary condition (OBC), while is unique under closed boundary condition (CBC).

\section{Open boundary condition}

Taking advantage of the spin-charge separation in the bosonized low-energy field theory, here we show that the 
condensation of $\theta_{s_{1,2}}$ opens a gap in the spin channel and gives rise to fourfold ground state degeneracy under OBC, which verifies that there is a spin- $1 / 2$ edge state at each boundary.

We first make a general statement: if the fields $2 \Theta_{1,2}$ condense, the ground states of the two spin channels are fourfold degenerate under $O B C$, which can be decomposed into a $\mathrm{SO}(3)$ singlet and a $\mathrm{SO}(3)$ triplet.

The condensation of $2 \Theta_{1}$ and $2 \Theta_{2}$, which is equivalent to the condensation of $\theta_{s_{1}}$ and $\theta_{s_{2}}$, suggests that the two spin channels are gapped. For simplicity, we consider the case that $2 \Theta_{1,2}$ condenses at value $0 \bmod 2 \pi$, i.e., $\Theta_{1,2}$ take 0 or $\pi \bmod 2 \pi$, namely $\left|\Theta_{1,2}\right\rangle=|0\rangle$ or $|\pi\rangle$. With the $\operatorname{SO}(3)$ symmetry, the fermion parities of the three species of fermions are all good quantum numbers. Since $\theta_{\alpha}$ shifts to $\theta_{\alpha}+\pi$ under the fermion parity operation $(-1)^{N_{\alpha}}$ of the $\psi_{\alpha}$ fermion, only the superposition states of $\Theta_{1,2}, \Theta_{1,2}^{ \pm}=|0\rangle \pm|\pi\rangle$, have a well-defined quantum number of $(-1)^{N_{\alpha}}$, which implies that the fourfold degenerate states in the spin channels in the thermodynamic limit are the following superposition states:

$$
\left|\Psi_{1,2,3,4}\right\rangle=\left|\Theta_{1}^{ \pm}\right\rangle \otimes\left|\Theta_{2}^{ \pm}\right\rangle \text {. }
$$

Then we show that these four states can be decomposed into an $\mathrm{SO}(3)$ singlet and an $\mathrm{SO}(3)$ triplet. As it is not convenient to study the $\mathrm{SO}(3)$ transformation on the bosonized fields, we consider its subgroup $\mathbb{Z}_{2} \times \mathbb{Z}_{2}$ which can protect the same nontrivial bosonic Haldane phase as $\mathrm{SO}(3)$ symmetry. We choose the two generators, denoted as $X$ and $Y$, to be the $\pi$ rotation around the $x$ and $y$ axes, i.e., under $X$, $\psi_{x} \rightarrow \psi_{x}, \psi_{y, z} \rightarrow-\psi_{y, z}$; under $Y, \psi_{y} \rightarrow \psi_{y}, \psi_{x, z} \rightarrow-\psi_{x, z}$. This indicates that under $X, \theta_{x} \rightarrow \theta_{x}, \theta_{y, z} \rightarrow \theta_{y, z}+\pi$ and under $Y, \theta_{y} \rightarrow \theta_{y}, \theta_{x, z} \rightarrow \theta_{x, z}+\pi$ while all $\phi$ fields remain unchanged. Therefore, in the ground-state space expanded by $\left|\Psi_{1,3,4,2}\right\rangle$, these operators are represented by

$$
\begin{aligned}
X & =\left(\begin{array}{cccc}
1 & 0 & 0 & 0 \\
0 & 1 & 0 & 0 \\
0 & 0 & -1 & 0 \\
0 & 0 & 0 & -1
\end{array}\right), \quad Y=\left(\begin{array}{cccc}
1 & 0 & 0 & 0 \\
0 & -1 & 0 & 0 \\
0 & 0 & 1 & 0 \\
0 & 0 & 0 & -1
\end{array}\right), \\
Z & =\left(\begin{array}{cccc}
1 & 0 & 0 & 0 \\
0 & -1 & 0 & 0 \\
0 & 0 & -1 & 0 \\
0 & 0 & 0 & 1
\end{array}\right)
\end{aligned}
$$

where $Z=X Y$. Now we can see that $\left|\Psi_{1}\right\rangle$ is invariant under $\mathbb{Z}_{2} \times \mathbb{Z}_{2}$, indicating that it is a singlet. To show that $\left|\Psi_{2,3,4}\right\rangle$ are triplets, we make the basis transformation, $\left|\Psi_{3}\right\rangle=-1 / \sqrt{2}\left(\left|\Psi_{1}\right\rangle+i\left|\Psi_{-1}\right\rangle\right),\left|\Psi_{2}\right\rangle=1 / \sqrt{2}\left(\left|\Psi_{1}\right\rangle-\right.$ $\left.i\left|\Psi_{-1}\right\rangle\right),\left|\Psi_{4}\right\rangle=\left|\Psi_{0}\right\rangle$. Under the new bases, the operators become

$$
\begin{aligned}
X^{\prime} & =\left(\begin{array}{ccc}
0 & 0 & -1 \\
0 & -1 & 0 \\
-1 & 0 & 0
\end{array}\right), \quad Y^{\prime}=\left(\begin{array}{ccc}
0 & 0 & 1 \\
0 & -1 & 0 \\
1 & 0 & 0
\end{array}\right), \\
Z^{\prime} & =\left(\begin{array}{ccc}
-1 & 0 & 0 \\
0 & 1 & 0 \\
0 & 0 & -1
\end{array}\right) .
\end{aligned}
$$

This are exactly the usual matrices of rotation operators $X, Y, Z$ in the triplet space in the $S_{z}$ representation. Therefore, we conclude that these three states $\left|\Psi_{2,3,4}\right\rangle$ form an $\operatorname{SO}(3)$ triplet. As the open chain has two ends, we can say that the fourfold degeneracy comes from the fact that, at every end point, there is a dangling spin $1 / 2$ which carries the projective representation of the $\mathrm{SO}(3)$ symmetry group and is thus protected. This statement can be further justified if we consider an additional reflection $Z_{2}$ symmetry seen in the density profile in Fig. 6.

In a one-dimensional open chain of length $L$, there may be nonvanishing tunneling between these four different degenerate states which leads to the energy splitting. Since the precise expression depends on the form of the Hamiltonian, we may take the bosonized form of the lattice model to evaluate the energy splitting. For instance, we can use the same method as in [30] to show that the energy splitting is proportional to $e^{-\alpha L}$, which will vanish in the thermodynamic limit.

\section{Closed boundary condition}

In contrast to the fourfold degeneracy under $\mathrm{OBC}$, we now show that the ground state is nondegenerate under $C B C$.

Since $\theta_{\alpha}(\alpha=x, y, z)$ shifts by $\pi$ under the fermion parity operation $(-)^{N_{\alpha}}$, the two possible pinned values $(0, \pi)$ of $\Theta_{1}$ are exchanged by either $(-)^{N_{x}}$ or $(-)^{N_{y}}$. Similarly, $\Theta_{2}$ would be exchanged under $(-)^{N_{y}}$ or $(-)^{N_{z}}$. Therefore, only the following four possible combinations of states are allowed:

$$
\left|\Psi_{1,2,3,4}\right\rangle=(|0\rangle \pm|\pi\rangle) \otimes(|0\rangle \pm|\pi\rangle) .
$$

As a result, in the ground-state space of the spin channels, we have the quantum numbers of parity of $(-)^{N_{x}+N_{y}},(-)^{N_{x}+N_{z}}$, and $(-)^{N_{y}+N_{z}}$ for the above four states as shown in Table I.

Now we show that, under $\mathrm{CBC}$, the ground state of the spin channels is nondegenerate. To preserve the $\mathrm{SO}(3)$ symmetry, there are only two different CBCs: periodic (PBC) and antiperiodic(APBC), namely, $\psi_{\alpha}(x+L)=\psi_{\alpha}(x)$ and $\psi_{\alpha}(x+$ $L)=-\psi_{\alpha}(x)$ for all $\alpha$.

In terms of the bosonized fields, the parity operators can be written as

$$
\begin{aligned}
& (-)^{N_{x}+N_{y}}=e^{i \sum_{\alpha=x, y} \phi_{\alpha}(L / 2)-\phi_{\alpha}(-L / 2)}, \\
& (-)^{N_{x}+N_{z}}=e^{i \sum_{\alpha=x, z} \phi_{\alpha}(L / 2)-\phi_{\alpha}(-L / 2)}, \\
& (-)^{N_{y}+N_{z}}=e^{i \sum_{\alpha=y, z} \phi_{\alpha}(L / 2)-\phi_{\alpha}(-L / 2)} .
\end{aligned}
$$

To have a uniform solution of $\Theta_{1}$ and $\Theta_{2}$ (the ground states are superpositions of uniform solutions), we must have

$$
\theta_{\alpha}(x+L)=\theta_{\alpha}(x)+2 n_{\alpha} \pi+\theta, \quad \alpha=x, y, z,
$$

where $n_{\alpha}$ is an integer and $\theta=0$ or $\pi$. Since $\Theta_{1}=\theta_{x}-\theta_{y}$ and $\Theta_{2}=\theta_{y}-\theta_{z}$, they do not depend on the value of $\theta$.

Consider the PBC case: $\psi_{R, L ; \alpha}(x+L)=\psi_{R, L ; \alpha}(x)$ and $\psi_{R, L ; \alpha}(x) \propto e^{-i\left( \pm \phi_{\alpha}-\theta_{\alpha}\right)} ; \phi_{\alpha}$ must satisfy

$$
\phi_{\alpha}(x+L)=\phi_{\alpha}(x)+2 n_{\alpha} \pi+\theta .
$$

Note that $\theta$ can only take 0 or $\pi$, otherwise $\Psi_{R, L}$ cannot satisfy the same boundary condition simultaneously. Now the aforementioned quantum numbers of the ground state should be $(-)^{N_{x}+N_{y}}=1,(-)^{N_{x}+N_{z}}=1$, and $(-)^{N_{y}+N_{z}}=1$, where we can see that only $\left|\Psi_{1}\right\rangle$ satisfies these quantum numbers. Therefore, if the system has the PBC, the ground state of the spin channel is $\left|\Psi_{1}\right\rangle=(|0\rangle+|\pi\rangle) \otimes(|0\rangle+|\pi\rangle)$ and is 
TABLE I. The quantum numbers of parity for the four possible ground states.

\begin{tabular}{ccccc}
\hline \hline & $\Psi_{1}=|+\rangle \otimes|+\rangle$ & $\Psi_{2}=|+\rangle \otimes|-\rangle$ & $\Psi_{3}=|-\rangle \otimes|+\rangle$ & +1 \\
\hline$(-1)^{N_{x}+N_{y}}$ & +1 & -1 & -1 & -1 \\
$(-1)^{N_{x}+N_{z}}$ & +1 & -1 & -1 & +1 \\
$(-1)^{N_{y}+N_{z}}$ & +1 & +1 & -1 & -1 \\
\hline \hline
\end{tabular}

nondegenerate, which is also true for APBC. Therefore, the spin channel of the ground state is unique under CBCs.

\section{APPENDIX B: ORDER PARAMETER}

With three species of fermion, we can construct all the parameters using the Gell-Mann matrice (in analog to the Pauli matrices for spin-1/2 fermions). The particle-hole channels are

$$
\Delta_{\mathrm{DW}}^{i}=\Psi_{R}^{\dagger} \gamma^{i} \Psi_{L}
$$

where $\tau^{i}(i=1,2, \ldots, 8)$ are the eight Gell-Mann matrices. $\Psi_{R, L}=\left(\psi_{R, L x}, \psi_{R, L y}, \psi_{R, L z}^{T}\right)$, where $\psi_{R, L \alpha}$ are the right- and left-moving fermions of the $\alpha$ species. Similarly, the particleparticle channels are

$$
\Delta_{\mathrm{SC}}^{i}=\Psi_{R} \gamma^{i} \Psi_{L}
$$

Using the bosonization identity

$$
\psi_{R \alpha}(x)=\frac{\eta_{\alpha}}{\sqrt{2 \pi \alpha}} e^{i k_{F} x} e^{-i\left(\phi_{\alpha}-\theta_{\alpha}\right)}
$$

and

$$
\psi_{L \alpha}(x)=\frac{\eta_{\alpha}}{\sqrt{2 \pi \alpha}} e^{-i k_{F} x} e^{-i\left(-\phi_{\alpha}-\theta_{\alpha}\right)},
$$

and choosing the eight Gell-mann matrices as

$$
\begin{aligned}
& \gamma^{1}=\left(\begin{array}{lll}
0 & 1 & 0 \\
1 & 0 & 0 \\
0 & 0 & 0
\end{array}\right), \quad \gamma^{2}=\left(\begin{array}{ccc}
0 & -i & 0 \\
i & 0 & 0 \\
0 & 0 & 0
\end{array}\right), \quad \gamma^{3}=\left(\begin{array}{ccc}
1 & 0 & 0 \\
0 & -1 & 0 \\
0 & 0 & 0
\end{array}\right), \quad \gamma^{4}=\left(\begin{array}{lll}
0 & 0 & 1 \\
0 & 0 & 0 \\
1 & 0 & 0
\end{array}\right), \\
& \gamma^{5}=\left(\begin{array}{ccc}
0 & 0 & -i \\
0 & 0 & 0 \\
i & 0 & 0
\end{array}\right), \quad \gamma^{6}=\left(\begin{array}{ccc}
0 & 0 & 0 \\
0 & 0 & 1 \\
0 & 1 & 0
\end{array}\right), \quad \gamma^{7}=\left(\begin{array}{ccc}
0 & 0 & 0 \\
0 & 0 & -i \\
0 & i & 0
\end{array}\right), \quad \gamma^{8}=\frac{1}{\sqrt{3}}\left(\begin{array}{ccc}
1 & 0 & 0 \\
0 & 1 & 0 \\
0 & 0 & -2
\end{array}\right) \text {, }
\end{aligned}
$$

the order parameters become

$$
\begin{gathered}
\Delta_{\mathrm{DW}}^{1}=\psi_{R x}^{\dagger} \psi_{L y}+\psi_{R y}^{\dagger} \psi_{L x}=\frac{\eta_{x} \eta_{y}}{2 \pi \alpha} e^{-i 2 k_{F} x} 2 i \sin \left(\theta_{y}-\theta_{x}\right) e^{i\left(\phi_{x}+\phi_{y}\right)}, \\
\Delta_{\mathrm{SC}}^{1}=\psi_{R x} \psi_{L y}+\psi_{R y} \psi_{L x}=\frac{\eta_{x} \eta_{y}}{2 \pi \alpha} 2 i \sin \left(\phi_{y}-\phi_{x}\right) e^{i\left(\theta_{x}+\theta_{y}\right)} \\
\Delta_{\mathrm{DW}}^{2}=-i\left[\psi_{R x}^{\dagger}(x) \psi_{L y}-\psi_{R y}^{\dagger} \psi_{L x}\right]=\frac{-2 i \eta_{x} \eta_{y}}{2 \pi \alpha} e^{-i 2 k_{F} x} \cos \left(\theta_{y}-\theta_{x}\right) e^{i\left(\phi_{x}+\phi_{y}\right)}, \\
\Delta_{\mathrm{SC}}^{2}=-i\left(\psi_{R x} \psi_{L y}-\psi_{R y} \psi_{L x}\right)=\frac{-2 i \eta_{x} \eta_{y}}{2 \pi \alpha} \cos \left(\phi_{y}-\phi_{x}\right) e^{i\left(\theta_{x}+\theta_{y}\right)} \\
\Delta_{\mathrm{DW}}^{3}=\left(\psi_{R x}^{\dagger} \psi_{L x}-\psi_{R y}^{\dagger} \psi_{L y}\right)=\frac{1}{2 \pi \alpha} e^{-i 2 k_{F} x}\left[e^{i 2 \phi_{x}}-e^{i 2 \phi_{y}}\right] \\
\Delta_{\mathrm{DW}}^{3}=\psi_{R x}^{\dagger} \psi_{L z}+\psi_{R x}^{\dagger} \psi_{L x}-\psi_{R x}=\frac{\eta_{x} \eta_{z}}{2 \pi \alpha} e^{-i 2 k_{F} x} 2 i \sin \left(\theta_{z}-\theta_{x}\right) e^{i\left(\phi_{x}+\phi_{z}\right)} \\
\Delta_{\mathrm{SC}}^{4}=\psi_{R x} \psi_{L z}+\psi_{R z} \psi_{L x}=\frac{\eta_{x} \eta_{z}}{2 \pi \alpha} 2 \sin \left(\phi_{z}-\phi_{x}\right) e^{i\left(\theta_{x}+\theta_{z}\right)} \\
\Delta_{\mathrm{DW}}^{5}=-i\left(\psi_{R x}^{\dagger} \psi_{L z}-\psi_{R z}^{\dagger} \psi_{L x}\right)=\frac{-2 i \eta_{x} \eta_{z}}{2 \pi \alpha} e^{-i 2 k_{F} x} \cos \left(\theta_{z}-\theta_{x}\right) e^{i\left(\phi_{x}+\phi_{z}\right)} \\
\Delta_{\mathrm{SC}}^{5}=-i\left(\psi_{R x} \psi_{L z}-\psi_{R z} \psi_{L x}\right)=\frac{-2 i \eta_{x} \eta_{z}}{2 \pi \alpha} \cos \left(\phi_{z}-\phi_{x}\right) e^{i\left(\theta_{x}+\theta_{z}\right)} \\
\Delta_{\mathrm{DW}}^{6}=\psi_{R y}^{\dagger} \psi_{L z}+\psi_{R z}^{\dagger} \psi_{L y}=\frac{\eta_{y} \eta_{z}}{2 \pi \alpha} e^{-i 2 k_{F} x} 2 i \sin \left(\theta_{z}-\theta_{y}\right) e^{i\left(\phi_{y}+\phi_{z}\right)} \\
\Delta_{\mathrm{SC}}^{6}=\psi_{R y} \psi_{L z}+\psi_{R z} \psi_{L y}=\frac{\eta_{y} \eta_{z}}{2 \pi \alpha} 2 i \sin \left(\phi_{z}-\phi_{y}\right) e^{i\left(\theta_{y}+\theta_{z}\right)} \\
2 \pi \alpha \\
\left.\Delta_{R y}^{\dagger} \psi_{L z}-\psi_{R z}^{\dagger} \psi_{L y}\right)=\frac{-i \eta_{y} \eta_{z}}{2 \pi \alpha k_{F} x} 2 \cos \left(\theta_{z}-\theta_{y}\right) e^{i\left(\phi_{y}+\phi_{z}\right)},
\end{gathered}
$$




$$
\begin{gathered}
\Delta_{\mathrm{SC}}^{7}=-i\left(\psi_{R y} \psi_{L z}-\psi_{R z} \psi_{L y}\right)=\frac{-i \eta_{y} \eta_{z}}{2 \pi \alpha} 2 \sin \left(\phi_{z}-\phi_{y}\right) e^{i\left(\theta_{y}+\theta_{z}\right)}, \\
\Delta_{\mathrm{DW}}^{8}=\psi_{R x}^{\dagger}(x) \psi_{L x}(x)+\psi_{R y}^{\dagger} \psi_{L y}-2 \psi_{R z}^{\dagger} \psi_{L z}=\frac{1}{2 \pi \alpha} e^{-i 2 k_{F} x}\left[e^{i 2 \phi_{x}}+e^{i 2 \phi_{y}}-2 e^{i 2 \phi_{z}}\right], \\
\Delta_{\mathrm{SC}}^{8}=\psi_{R x} \psi_{L x}+\psi_{R y} \psi_{L y}-2 \psi_{R z} \psi_{L z}=\frac{1}{2 \pi \alpha}\left[e^{i 2 \theta_{x}}+e^{2 i \theta_{y}}-2 e^{2 i \theta_{z}}\right], \\
\Delta_{\mathrm{DW}}=\psi_{R x}^{\dagger} \psi_{L x}+\psi_{R y}^{\dagger} \psi_{L y}+\psi_{R z}^{\dagger} \psi_{L z}=\frac{1}{2 \pi \alpha} e^{-i 2 k_{F} x}\left[e^{i 2 \phi_{x}}+e^{i 2 \phi_{y}}+e^{i 2 \phi_{z}}\right], \\
\Delta_{\mathrm{SC}}=\psi_{R x} \psi_{L x}+\psi_{R y} \psi_{L y}+\psi_{R z} \psi_{L z}=\frac{1}{2 \pi \alpha}\left[e^{i 2 \theta_{x}}+e^{2 i \theta_{y}}+e^{2 i \theta_{z}}\right],
\end{gathered}
$$

where the last two quantities are related to the identity matrix and both areSO(3) invariant.

Now we discuss the dominant correlation function for the doped fermionic Haldane phase where $\phi_{c}$ is gapless while $\theta_{s_{1}}$ and $\theta_{s_{2}}$ are condensed (i.e., $2 \theta_{x}-2 \theta_{y}, 2 \theta_{y}-2 \theta_{z}$ are condensed, therefore $2 \theta_{y}-2 \theta_{z}$ is also condensed). Therefore $\phi_{s_{1}}, \phi_{s_{2}}$ are disordered and exponentially decaying, which indicates that $2 \phi_{x}-2 \phi_{y}, 2 \phi_{y}-2 \phi_{z}, 2 \phi_{y}-2 \phi_{z}$ are disordered and exponentially decaying. Then $\Delta_{\mathrm{DW}}^{i}$ and $\Delta_{\mathrm{SC}}^{i}(i \neq 3,8)$ are all exponentially decaying. Due to the $\mathrm{SO}(3)$ symmetry, $\Delta_{\mathrm{DW}}^{3,8}$ and $\Delta_{S C}^{3,8}$ are vanishing. Now only the $\mathrm{SO}(3)$-singlet superconductivity order parameters $\Delta_{\mathrm{SC}}$ are power-law decaying: $\Delta_{\mathrm{SC}}\left(r+r_{0}\right) \Delta_{\mathrm{SC}}\left(r_{0}\right) \propto 1 / r^{2 /\left(3 K_{c}\right)}$.

On the other hand, if the spin channels are gapped by condensing $2 \phi_{x}-2 \phi_{y}$ and $2 \phi_{y}-2 \phi_{z}$, then $\Delta_{\mathrm{SC}}$ are exponentially decaying but $\Delta_{\mathrm{DW}}$ are power-law decaying: $\Delta_{\text {DW }}\left(r+r_{0}\right) \Delta_{\text {DW }}\left(r_{0}\right) \propto 1 / r^{\frac{2 K_{c}}{3}}$. In addition to this $2 k_{F}$ part contribution, there is also a zero momentum part, namely $\Delta_{\text {DW }}^{\prime}=\sum_{\alpha} n_{\alpha, R}+n_{\alpha, L} \propto \partial \phi_{c}$, which decays faster than the $2 k_{F}$ part since its power is $2 K_{c}$.

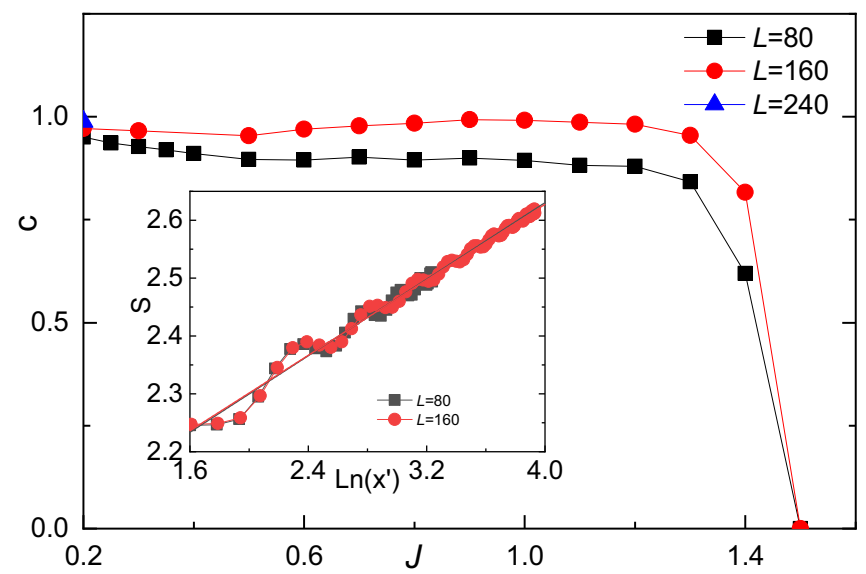

FIG. 5. The extracted central charge $c$ for the model in Eq. (2) (in the main text) at doping level $\delta=5 \%$ and $U=4$ as a function of $J$. Inset: von Neumann entanglement entropy $S$ at $U=4$ and $J=0.3$, where $x^{\prime}=\frac{L}{\pi} \sin \left(\frac{x \pi}{L}\right)$ and the linear line is the fit to $S=\frac{c}{6} \ln \left(x^{\prime}\right)+\tilde{c}$.

\section{APPENDIX C: DETERMINATION OF THE PHASE DIAGRAM}

\section{Central charge and the phase boundary}

Upon doping, if $J$ is not very large, the ground state of the system becomes superconducting with one gapless charge mode which is the $c=1$ Luttinger liquid. To confirm this, we calculate the von Neumann entanglement entropy. In the DMRG simulation, the central charge can be obtained by calculating the von Neumann entropy $S=-\operatorname{Tr} \rho \ln \rho$, where $\rho$ is the reduced density matrix of a subsystem with length $x$. For a critical system, it has been established [31] that $S(x)=$ $\frac{c}{6} \ln \left[\frac{L}{\pi} \sin \left(\frac{x \pi}{L}\right)\right]+\tilde{c}$ for an open system, where $c$ is the central charge of the conformal field theory and $\tilde{c}$ denotes a model dependent constant. We use the central charge to determine the phase diagram (Fig. 1 in the main text) in the main text. For typical parameters, our results, shown in Fig. 5, show that the central charge is $c=1$ when $J<1.45$, consistent with one gapless charge mode while both spin modes are gapped (which only shift $\tilde{c}$ by some constant).

\section{Charge density profile and phase separation}

Here we will briefly discuss the phase separation (PS) region. As shown in Fig. 6 for $J / t=1.6$ in the PS regime, the charge distribution is nonhomogeneous: while some parts of the system are full of particles, the remaining parts of

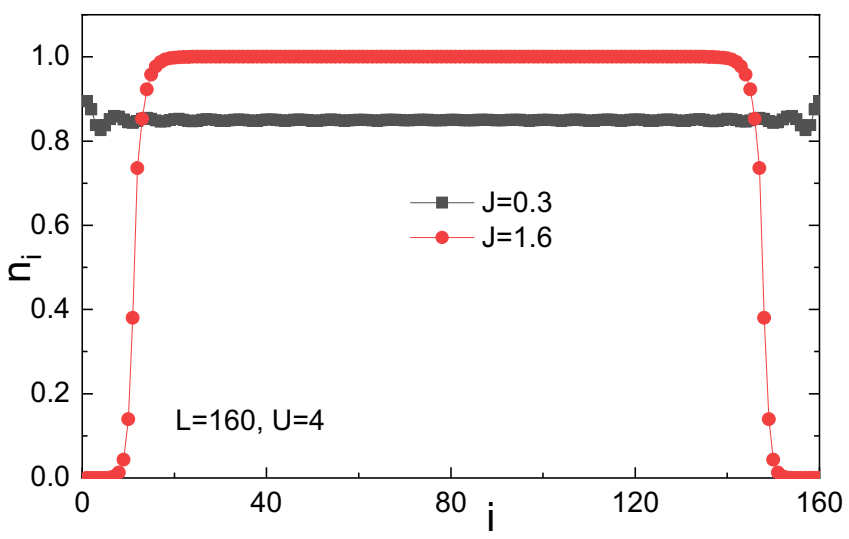

FIG. 6. The density profile $n_{i}$ in the superconducting (black) and phase separation (red) regions. Here, $U=4$. 
the system are empty without any particle, e.g., $n_{i} \sim 0$ close to the boundaries. This indicates that the doped holes are accumulated in the regions close to the boundaries, while the middle regime is at $1 / 3$ filling which turns out to be in the fully gapped SPT phase [20]. The PS phenomenon was also present in the $t-J$ model(with projection) [32,33]. For comparison, we also show the density profile in the SC regime in Fig. 6, where a homogeneous charge distribution is seen despite the small charge oscillation caused by the boundary effect.

Intuitively, this PS can be understood by comparing the energy gain from the hopping holes, and the energy cost by breaking the spin valence bonds. The energy gain from hopping holes is around $t$, while the energy cost of breaking the spin valence bonds is around $J$. To understand this, we turn to the undoped case where there is only one particle per site if $U$ is large enough and hence the degree of freedom on every site is just spin 1 . When this spin 1 is in the Haldane phase, the structure of this state can be viewed as the famous AKLT (Affleck, Lieb, Kennedy, and Tasak) state [34] where the spins 1 are represented as two spins $1 / 2$ that project on the spin- 1 channel and two spins $1 / 2$ from two nearest-neighbor sites form a spin singlet. The characteristic energy of such a spin singlet is around $J$. However, in the presence of holes, the on-site degree of freedom there is no longer the spin one which would cause the characteristic energy cost of $J$. Therefore, when $J$ is large enough, the energy cost of the hopping hole overcomes the gain, which results in the phase separation. In other words, when the interactions that drive the bSPT phase at a certain filling are strong enough, they would repel the charge fluctuation, i.e., hole doping, in it.
[1] Z.-C. Gu and X.-G. Wen, Phys. Rev. B 80, 155131 (2009).

[2] C. L. Kane and E. J. Mele, Phys. Rev. Lett. 95, 146802 (2005).

[3] M. Z. Hasan and C. L. Kane, Rev. Mod. Phys. 82, 3045 (2010).

[4] X.-L. Qi and S.-C. Zhang, Rev. Mod. Phys. 83, 1057 (2011).

[5] F. Haldane, Phys. Lett. A 93, 464 (1983).

[6] F. D. M. Haldane, Phys. Rev. Lett. 50, 1153 (1983).

[7] T. Senthil and M. Levin, Phys. Rev. Lett. 110, 046801 (2013).

[8] X. Chen, Z.-X. Liu, and X.-G. Wen, Phys. Rev. B 84, 235141 (2011).

[9] X. Chen, Z.-C. Gu, Z.-X. Liu, and X.-G. Wen, Science 338, 1604 (2012).

[10] X. Chen, Z.-C. Gu, Z.-X. Liu, and X.-G. Wen, Phys. Rev. B 87, 155114 (2013).

[11] A. P. Schnyder, S. Ryu, A. Furusaki, and A. W. W. Ludwig, Phys. Rev. B 78, 195125 (2008).

[12] A. Kitaev, in Advances in Theoretical Physics: Landau Memorial Conference, 22-26 June 2008, Chernogolokova, Russia, edited by V. Lebedev and M. Feigel'man, AIP Conf. Proc. No. 1134 (AIP, New York, 2009), p. 22.

[13] L. Fidkowski and A. Kitaev, Phys. Rev. B 81, 134509 (2010).

[14] L. Fidkowski and A. Kitaev, Phys. Rev. B 83, 075103 (2011).

[15] Z.-C. Gu and X.-G. Wen, Phys. Rev. B 90, 115141 (2014).

[16] M. Cheng, N. Tantivasadakarn, and C. Wang, Phys. Rev. X 8, 011054 (2018).

[17] Q.-R. Wang and Z.-C. Gu, Phys. Rev. X 8, 011055 (2018).

[18] Q.-R. Wang and Z.-C. Gu, arXiv:1811.00536.
[19] C. Wang, C.-H. Lin, and Z.-C. Gu, Phys. Rev. B 95, 195147 (2017).

[20] S.-Q. Ning, H.-C. Jiang, and Z.-X. Liu, Phys. Rev. B 91, 241105(R) (2015).

[21] F. Pollmann, A. M. Turner, E. Berg, and M. Oshikawa, Phys. Rev. B 81, 064439 (2010).

[22] S. R. White, Phys. Rev. Lett. 69, 2863 (1992).

[23] R. Assaraf, P. Azaria, M. Caffarel, and P. Lecheminant, Phys. Rev. B 60, 2299 (1999).

[24] K. Buchta, O. Legeza, E. Szirmai, and J. Sólyom, Phys. Rev. B 75, 155108 (2007).

[25] A. Keselman, E. Berg, and P. Azaria, Phys. Rev. B 98, 214501 (2018).

[26] C. L. Kane, R. Mukhopadhyay, and T. C. Lubensky, Phys. Rev. Lett. 88, 036401 (2002).

[27] E. Lieb, T. Schultz, and D. Mattis, Ann. Phys. (NY) 16, 407 (1961).

[28] M. Cheng, M. Zaletel, M. Barkeshli, A. Vishwanath, and P. Bonderson, Phys. Rev. X 6, 041068 (2016).

[29] P. W. Anderson, Science 235, 1196 (1987).

[30] L. Fidkowski, R. M. Lutchyn, C. Nayak, and M. P. A. Fisher, Phys. Rev. B 84, 195436 (2011).

[31] P. Calabrese and J. Cardy, J. Stat. Mech. (2004) P06002.

[32] M. Ogata, M. U. Luchini, S. Sorella, and F. F. Assaad, Phys. Rev. Lett. 66, 2388 (1991).

[33] Q.-R. Wang and P. Ye, Phys. Rev. B 90, 045106 (2014).

[34] I. Affleck, T. Kennedy, E. H. Lieb, and H. Tasaki, Phys. Rev. Lett. 59, 799 (1987). 